\title{
Les figures de la gestion du changement sociotechnique
}

\section{Figures that manage sociotechnical change}

\author{
François-Xavier de Vaujany *
}

\begin{abstract}
Résumé
Les outils informatiques occupent une place grandissante dans la vie des organisations. L'étude des dynamiques sociales liées à leurs usages est ainsi devenue une préoccupation importante pour les sciences sociales. L'article qui suit présente une synthèse des travaux récents sur cette thématique, plus particulièrement sur les recherches structurationnistes. Un modèle fédérateur est suggéré : le modèle archétypique. Celui-ci correspond à trois situations stylisées du changement sociotechnique (archétype neutre, régénéré et perturbé) pouvant, dans certains cas, être mises bout à bout afin de reconstituer des trajectoires appropriatives. Le cadre théorique ainsi élaboré est appliqué à différentes technologies de l'information, notamment des progiciels de gestion intégrés (PGI), des intranets ou des systèmes type messagerie. L'article s'efforce ensuite d'aller plus loin que le simple stade de la compréhension des dynamiques sociales en articulant les trajectoires appropriatives avec des logiques de contrôle qui leurs seraient spécifiques. Ces logiques correspondent à des figures de gestionnaires (le canalisateur, le facilitateur et le catalyseur) qui sont précisées dans cette recherche.
\end{abstract}

\begin{abstract}
Computers and software occupy an ever bigger place in an organization's activities. Studying the social dynamics of their uses has thus become a major preoccupation for the social sciences. An overview of recent studies, in particular "structurationist" research, on this subject is presented; and a federative model, suggested. This archetypical model corresponds to three stylized situations of sociotechnical change (a neutral, regenerated and disturbed archetype) that, in some cases, can be aligned to reconstitute "appropriative trajectories". This theoretical framework is applied to various sorts of information technology, in particular: enterprise resource planning (ERP) software, intranets and electronic messaging systems. Beyond merely understanding the social dynamics, the effort is
\end{abstract}

\footnotetext{
* Earlier draft of an article published in Sociologie du travail 45 (2003) 515-536
} 
made to link appropriative trajectories with the "logics of control" specific to them. The latter correspond to figures of management (the channel, facilitator and catalyst), which are described.

Mots clés : Changement sociotechnique ; Technologies de l'information ; Modèle archétypique ; Trajectoires appropriatives ; Structuration; A. Mallard

Keywords: Sociotechnical change; Archetypical model; Appropriative trajectories; Structurationism; Information age technology; A. Mallard

Le courant sociotechnique correspond à une perspective de recherche vieille de plus d'une cinquantaine d'années. Les premiers objets sur lesquels il s'est penché avaient trait aux productions minières ou industrielles. Lors d'une recherche-action devenue célèbre, Eric Trist et Ken Bamforth s'étaient intéressés à l'effet de la mécanisation de l'abattage du charbon dans les mines anglaises. La mise en place de nouvelles modalités de constitution et de gestion des équipes, très standardisées, avait amené une différenciation plus marquée des individus, des qualifications accentuées et une organisation du travail plus rigide... qui coïncidèrent avec un climat social détérioré. Constatant l'inadéquation évidente entre la technologie d'abattage standardisée et les habitudes des mineurs, E. Trist avança alors l'idée que l'organisation formait un système sociotechnique et que l'amélioration des conditions du travail passait par un travail simultané sur le système social et sur la technologie, un principe d' « optimisation-jointe » (Trist et Bamforth, 1951 ; Emery et Trist, 1969) ${ }^{1}$. Durant les deux décennies qui suivirent, avec l'institutionnalisation des travaux du Tavistock Institute, les modèles s'affinèrent. Ils intégrèrent davantage de variables environnementales au système sociotechnique (Emery et Trist, 1973 ; Shani et al., 1992).

C'est dans les années 1980 que l'intérêt des chercheurs en sciences sociales en général, et en gestion en particulier, s'est porté sur le cas des technologies informatiques. Après une période de dichotomisation du système sociotechnique, avec des visions oscillant entre déterminisme technologique et déterminisme organisationnel (Pasmore et al., 1982 ; Desanctis et Poole, 1994 ; Yong-Young, 2000), la perspective sociotechnique revient en force dans le domaine des technologies de l'information. Que ce soit avec l'approche structurationniste et les travaux de Stephen R. Barley (Barley, 1986), Wanda J. Orlikowski (Orlikowski, 1992 ; Orlikowski, 2000), W.J. Orlikowski et Dan Robey (Orlikowski et Robey, 1991), Gerardine Desanctis et Marshall S. Poole (Desanctis et Poole, 1992 ; Desanctis et Poole, 1994), Hamid Bouchikhi (Bouchikhi, 1990), Naklé El Haddad (El Haddad, 1987) ou encore Geoff Washam (Washam, 1993), avec les travaux relatifs à la sociologie des réseaux, notamment ceux de Pat Fung, Martin Lea et Tim O'Shea (Fung et al., 1995) ou encore les recherches marquées par l'approche réaliste critique, notamment les travaux de Philip J. Dobson (Dobson, 1999 ; Dobson, 2002) ou de François-Xavier de Vaujany (Vaujany, 2001b ; Vaujany, 2001c), les perspectives intégratives reprennent de leur vigueur. Une évolution profonde du monde de l'entreprise va les y aider.

\footnotetext{
${ }^{1}$ On remarque au passage que les préoccupations d'E. Trist n'avaient au début aucun caractère productiviste. D'après Enid Mumford : « à la différence des réorganisations d'aujourd'hui, les motivations à la base de l'approche du Tavistock Institute n'étaient pas d'augmenter la productivité, mais de réduire le stress et de donner un environnement de travail pour les mineurs. Le fait que la production augmente une fois le nouveau système introduit était un bénéfice inattendu et une bonne surprise » (Mumford, 1999, p. X).
} 
Dans les processus de soutien administratif, dans les activités de production industrielle, dans les activités de traitement ou de production de service, les outils informatiques médiatisent, remplacent ou supportent un nombre croissant d'activités. Le temps passé devant des applications informatiques prend une part grandissante de la durée du travail. Pour le cas de la France, de 1987 à 1998, le nombre de salariés utilisateurs d'outils informatiques est passé de 24 à $51 \%$ de la population totale (Cézard et al., 2000). Parallèlement, le temps passé devant des ordinateurs a lui aussi explosé. En moyenne (utilisateurs et non-utilisateurs confondus),

« une heure de travail sur cinq se passe désormais sur ordinateur », et " la moitié des utilisateurs sont devant leur écran plus de trois heures par jour» (Cézard et al., 2000). Ces chiffres peuvent même être beaucoup plus élevés en fonction des catégories de travailleurs étudiées puisqu'une étude réalisée sur plus de 1000 salariés d'un grand groupe a montré que les employés de bureau consacraient près de la moitié de leur emploi du temps $(46 \%)$ à travailler sur leur micro-ordinateur (Legrenzi, 1997).

Durant les dix dernières années, l'océan de l'informatique de réseau a envahi les entreprises, porté par plusieurs vagues successives : les messageries électroniques, l'EDI (échange de données informatisées), les collecticiels, les intranets, et plus récemment, les PGI (progiciels de gestion intégrés). Ces derniers occupent également une part croissante de l'emploi du temps des salariés.

Dans le cadre de l'article qui suit, une grille d'analyse sociotechnique est suggérée. Elle est directement inspirée des travaux structurationnistes et suggère des éléments de réponse à la question suivante : comment gérer le changement sociotechnique en système d'information à partir de dynamiques de contrôle les plus évolutives possibles ?

La première partie reprend diverses contributions structurationnistes en les résumant par un modèle global : le modèle archétypique (1). Celui-ci permettra de mieux saisir les dynamiques liées à l'utilisation des technologies de réseau. La seconde partie développe des concepts-clés de la gestion du changement sociotechnique, intégrés sous forme de trois configurations de gestion. Elles sont ensuite articulées avec trois figures de gestionnaire : le canalisateur, le facilitateur et le catalyseur (2).

\section{Un modèle archétypique du changement sociotechnique}

Le modèle présenté dans cette première partie est construit à partir de différents travaux structurationnistes. Cette approche est née au milieu des années 1980. Elle s'appuie sur les recherches d'Anthony Giddens (Giddens, 1979 ; Giddens, 1984), Jean Piaget (Piaget, 1947 ; Piaget, 1977), Margaret S. Archer (Archer, 1982 ; Archer, 1995) ou encore Roy Bhaskar (Bhaskar, 1979) afin de modéliser les interactions organisation-technologie d'un point de vue processuel. Dans le cas du système d'information, l'objet du structurationnisme est de comprendre comment des interactions médiatisées ou supportées par la technologie vont reproduire ou produire les structures sociales de l'organisation. Globalement, le courant de recherche peut-être ramené à deux sous-courants constituant davantage les deux extrêmes d'un continuum que des écoles de pensée à part entière. En effet, si certains travaux sont relativement fidèles au principe de non-extériorité des structures défendu par la théorie de la structuration, d'autres adoptent une position théorique radicalement différente en considérant que la technologie peut avoir sur certaines régions une influence indépendante des médiations ou interactions sociales (Jones, 1999 ; Vaujany, 
2001a) ${ }^{2}$. Par rapport à la segmentation du champ, le modèle archétypique qui suit présente un avantage certain, puisqu'il peut s'interpréter tant du point de vue interprétatif d'A. Giddens, pour lequel les structures ne sont que des «traces mnésiques » dans la tête des acteurs, que du point de vue réaliste critique ${ }^{3}$ qui accorde une certaine matérialité et une puissance de contrainte aux structures.

\subsection{Les fondamentaux du modèle}

Le « modèle archétypique » proposé correspond à un travail de synthèse des recherches structurationnistes. Il ramène en fait les dynamiques sociales liées à l'usage des technologies de l'information à trois cas extrêmes, lesquels serviront de point d'ancrage au modèle archétypique. Ces situations stylisées, les archétypes technologiques, sont des « états structurationnels, des configurations sociotechniques durables décrites au travers de dimensions structurantes » (Vaujany, 2001b ; Vaujany, 2001c). La première des situations archétypiques correspond à l'archétype neutre $(\mathrm{N})$. Dans cette première configuration, soit le système n'est pas utilisé, soit il l'est, mais d'une façon qui reproduit et renforce largement les modes de fonctionnement de l'organisation. La technologie ne fait que se dissoudre dans des routines qui préexistaient à la mise en œuvre du nouveau système. La littérature structurationniste montre que cette première dynamique est largement dominante. Souvent, l'innovation technologique ne fait que s' « enkyster » dans les modes d'interaction habituels, même lorsqu'elle a été pensée de façon à encourager le changement. C'est le cas par exemple d'une application de gestion des ressources humaines sous intranet qui correspondait auparavant à une application Minitel. Les employés ont finalement utilisé l'outil de façon proche de l'ancien, en négligeant les nouvelles fonctionnalités et en continuant à utiliser, pour la gestion des congés payés, d'anciens circuits de validation (Vaujany, 2001c). La seconde situation prend la forme de l'archétype régénéré (R). Les usages du nouvel outil ont alors un rôle régénérant pour le système social. L'organisation devient le terrain de véritables innovations sociales à l'usage. De nouvelles routines organisationnelles émergent en liaison directe avec la technologie. Un exemple typique est celui de la mise en œuvre d'un nouveau système de messagerie dans un service de recherche-développement. Les personnels impliqués ont alors élaboré de toutes nouvelles méthodes de recherche d'information, plus transversales. Ils ont commencé à communiquer davantage avec des universités étrangères ainsi qu'avec d'autres centres de recherche du groupe. De nouvelles collaborations ont pu être lancées et le système de rôles du service a connu une évolution profonde (Vaujany, 1999). La troisième situation, l'archétype perturbé $(\mathrm{P})$ correspond à l'émergence de conflits de natures diverses au sein de l'organisation. L'archétype peut se superposer aux deux précédents. Les usages ont alors un rôle déstructurant par rapport aux routines organisationnelles. Dans certains cas, la mise en œuvre de la technologie peut aboutir à l'interruption des processus à l'œuvre dans certaines

\footnotetext{
${ }^{2}$ D’après Matthew Jones : « Comparé à la théorie de la structuration adaptative, il devrait être évident que le modèle de W.J. Orlikowski est plus proche de l'esprit de la théorie de la structuration telle que l'a décrite A. Giddens [...] » (Jones, 1999, p. 126).

3 Pour une présentation générale du réalisme critique, on pourra consulter John Mingers (Mingers, 2000). La lecture de Geoffrey Hodgson (Hodgson, 1999) pourra également aider le lecteur à mieux situer le réalisme critique par rapport à la théorie de la structuration et à l'institutionnalisme.
} 
régions. Un PGI implanté dans une entreprise commerciale peut ainsi amener la suspension du processus de saisie et de traitement des commandes. Des vendeurs peuvent en effet juger que le nouvel outil renforce le contrôle central. S'il n'est pas possible d'entrer dans des stratégies d'évitement, la situation peut déboucher sur une impasse. La saisie des commandes est alors tout simplement interrompue. L'archétype perturbateur, dans cette situation spécifique, correspond alors à un archétype per se.

Pour revenir sur les racines sociologiques de l'approche structurationniste, on remarque que l'on peut faire sens des trois archétypes tant du point de vue interprétatif que dans l'optique du réaliste critique.

Dans la perspective d'A. Giddens, chacun des trois archétypes va correspondre à une situation psychosociologique spécifique. Dans le premier cas (N), ce qu'A. Giddens appelle le sentiment de « sécurité ontologique » des acteurs, c'est-à-dire leur sentiment de continuité et de confiance par rapport à leurs actes, est maintenu. Les utilisations ne font qu'actualiser des propriétés du structurel déjà présentes dans l'esprit des membres de l'organisation. Dans le cas de l'archétype perturbateur, le sentiment de sécurité ontologique est rompu. Cela se traduit par des angoisses fortes pour les acteurs ${ }^{4}$. Les utilisations peuvent actualiser ou produire des structures sociales, mais quoi qu'il en soit, le processus est particulièrement angoissant pour les personnes concernées. Pour la dernière situation (R), les usages sont producteurs de nouvelles structures sociales. L'instanciation est innovante. Dans l'esprit des acteurs, le système de rôles évolue en profondeur. S'il a été rompu lors d'une phase précédente, le sentiment de sécurité ontologique est en voie de reconstruction.

Selon le modèle de M.S. Archer, les trois archétypes sont des états sociaux ayant des caractéristiques plus objectivables que pour A. Giddens ; structures et actions sont à dissocier. M.S. Archer considère que les structures (notamment techniques) précèdent et conditionnent l'action. Dans le cas de l'archétype neutre, le pouvoir conditionnant des structures est supérieur à celui des actions, notamment des usages. Les utilisations de la technologie reproduisent le système de rôles ainsi que les cloisonnements organisationnels qui lui sont associés. Pour une situation « régénérée » $(\mathrm{R})$, la situation est tout autre. Les actions sont plus fortes que le pouvoir conditionnant des structures et les usages transforment le système de rôles en place avant le déploiement du nouveau système technique. Ce dépassement du pouvoir conditionnant des structures peut reposer soit sur l'acceptation d'un coût (matériel, symbolique ou psychologique), soit sur la capacité d'inventivité des utilisateurs pour « contourner » les contraintes structurelles. Enfin, dans une situation perturbée, actions et structures entrent en tension. Cela se traduit par l'émergence d' « incompatibilités » au sein du système de rôles.

Afin d'instrumenter les archétypes, nous suggérons de reprendre la voie proposée par S.R. Barley (Barley, 1986 ; Barley, 1990) basée sur l'étude du système de rôles ${ }^{5}$, objet qui pourra être étudié via les descripteurs suivants :

\footnotetext{
4 On remarque au passage que le lien entre structure et anxiété, central dans la théorie de la structuration, est aussi fondamental dans les travaux du Tavistock Institute, en particulier ceux d'Elliot Jacques (Jacques, 1957).

5 D'après S.R. Barley, si l' « on conçoit les structures comme étant une forme globale qui émerge à partir des relations existant entre les membres d'une collectivité, alors il est possible de relier directement et empiriquement un changement dans le système de rôles à une modification structurelle en examinant les propriétés des réseaux sociaux » (Barley, 1990, p. 69). Afin d'instrumenter une évolution du système de rôles et donc des structures
} 
- le périmètre et la densité du système de rôles (nombre d'éléments en interaction, degré d'ouverture sur l'environnement, degré d'interdépendance) ;

- les formes d'interaction (fréquence d'interaction des agents, séquences d'interaction typiques, identification des éléments influents du processus);

- les identités réciproques, c'est-à-dire la façon dont les agents se définissent les uns par rapport aux autres dans le système de rôles.

La définition du système de rôles avancée est proche d'un positionnement réaliste critique. Le périmètre du système et sa structure d'interaction présentent des caractéristiques objectivables qui pourront influer sur les dynamiques d'appropriation indépendamment du projet et de la perception d'un ensemble d'acteurs donné. De leur point de vue, le système de rôles présente d'ailleurs une véritable multidimensionnalité qui pourrait bien être problématique. Heureusement, comme le souligne Erving Goffman (Goffman, 1967), une « séparation des rôles » est souvent permise par la «séparation des publics ». L'univers étudié n'est cependant pas de nature positive. Une place certaine est laissée aux interprétations ${ }^{6}$ et les processus sont considérés comme indéterminés même s'ils sont contraints.

\subsection{Le modèle archétypique en mouvement : les trajectoires appropriatives}

La présentation des trois archétypes précédents donne une vision relativement statique des systèmes sociotechniques. Afin de reconstituer les processus d'appropriation technologique dans leur durée, le recours à la notion de trajectoires appropriatives peut être utile. Il s'agit d'un « enchaînement régulier d'archétypes technologiques » pour un système sociotechnique donné (Vaujany, 2001b ; Vaujany, 2001c). Un processus sociotechnique pourra ainsi être décrit en fonction de la séquence archétypique qu'il intègre (exemple : N-P-N), du rythme de réalisation du changement archétypique (relativement à diverses dynamiques d'appropriation) et du degré de cohérence archétypique pour le groupe d'acteur ciblé.

Une analyse fouillée des dynamiques sociotechniques est développée par W.J. Orlikowski (Orlikowski, 2000). Dans un article de référence, l'auteur avance un modèle centré davantage sur l'innovation que l'inertie. D'après elle, la plupart des chercheurs en système d'information considèrent que la « technologie-en-pratique $»^{7}$, au terme d'une phase d'apprentissage, va se fondre dans des routines qui vont ensuite perdurer. Or, nombre de travaux, notamment ceux de Claudio U. Ciborra (Ciborra, 1997 ; Ciborra, 1999 ; Ciborra, 2001) abondent dans le sens de dynamiques plus continues et innovantes. D'après

sociales, S.R. Barley propose de s'intéresser aux « scripts », c'est-à-dire « aux formes récurrentes d'interaction qui définissent, dans des termes observables et comportementaux l'essence des rôles des acteurs » (Barley, 1986, p. 83, citant Schanck et Abelson, 1977).

6 Ce qui ne veut pas dire, conformément au modèle morphogénétique de M.S. Archer (Archer, 1995), que toutes les interprétations se valent. Certaines peuvent être « fausses » et constituer une erreur par rapport à un contexte de conditionnement non perçu par l'acteur. En outre, certains éléments du réel peuvent être l'objet d'interprétations « intransitives » d'un acteur à l'autre. Certains objets ou signes sont ainsi plus « interprétables » que d'autres.

${ }^{7}$ Une « technologie-en-pratique » est en fait « la structure spécifique activée de façon routinière lorsque nous utilisons une machine particulière, une technique, un outil ou un gadget de façon récurrente dans nos activités quotidiennes » (Orlikowski, 2000, p. 408). 
cette seconde perspective, les outils vont faire l'objet de multiples « bricolages » par les utilisateurs-finaux.

Nous allons commencer par présenter l' « anti-modèle » présent en filigrane dans l'article de W.J. Orlikowski (Orlikowski, 2000), que nous proposons de nommer la trajectoire du point d'équilibre, avant de présenter la trajectoire « improvisationnelle » suggérée par W.J. Orlikowski, en nous appuyant sur la grille archétypique introduite précédemment. Enfin, nous finirons par le développement d'une troisième dynamique, la trajectoire catalytique, relativement absente de la littérature structurationniste.

\subsubsection{La trajectoire du point d'équilibre}

Selon cette première dynamique, la routine et l'inertie sont des états courants en ce qui concerne les systèmes sociotechniques. L'archétype neutre est donc la configuration la plus souvent observée en matière d'usages informatiques. La trajectoire équilibrée peut-être décomposée en deux sous-trajectoires débouchant sur une innovation sociale à l'usage, l'une passant par une phase de perturbation, l'autre non.

Pour la première sous-trajectoire (N-P-R-N), le système sociotechnique commence par connaître une première phase où la technologie est utilisée de façon analogique. Les employés finalisent le nouvel outil à partir de schèmes d'utilisation similaires à ceux de technologies antérieures. Les routines organisationnelles sont maintenues voire renforcées par cette première étape. Dans la seconde phase, les utilisations traversent une porte symbolique (G1) qui marque l'interruption de l'action routinière. L'étape suivante correspond à l'archétype perturbé. Les utilisateurs sont conscients de la nouveauté du système qu'ils s'efforcent alors d'insérer de façon innovante dans l'organisation. Cette phase intermédiaire correspond au passage par une sorte de sas relativement déstructurant pour le système de rôles. Certains processus sont interrompus. D'autres sont simplement gênés dans leur déploiement. Cette situation pourra ensuite déboucher sur une véritable situation d'innovation sociale à l'usage lorsqu'une dernière porte symbolique (G2) sera franchie. Elle illustre le glissement de la situation perturbée (éventuellement régénérante) vers une situation d'innovation harmonieuse. Cette dernière pourra durer plus ou moins longtemps d'une organisation à l'autre ${ }^{8}$.

La deuxième sous-trajectoire $(\mathrm{N}-\mathrm{R}-\mathrm{N})^{9}$ est proche de la précédente à une exception près : le processus d'innovation sociale à l'usage n'implique pas le passage par une phase intermédiaire de perturbation. L'émergence de nouvelles routines organisationnelles se fait de façon harmonieuse avant qu'un nouvel état neutre ne s'installe.

Les exemples illustrant la trajectoire équilibrée sont multiples dans la littérature structurationniste. Ils montrent presque tous la difficulté des systèmes sociotechniques à fran-

\footnotetext{
8 Comment caractériser le passage d'une phase à l'autre ? Cette question est évidemment centrale. Tout dépendra du positionnement épistémologique choisi par le chercheur-accompagnant ou le consultant. Dans le strict prolongement d'A. Giddens, le passage de R à N par exemple sera fonction du ressenti des acteursfinaux, et de celui-ci uniquement. C'est lorsque la sensation d'innovation s'épuisera dans l'esprit des utilisateurs que l'on rentrera à nouveau dans une phase neutre. À l'inverse, selon les chercheurs optant pour un positionnement réaliste critique, le changement archétypique sera fonction d'évolution « objectivées » qui intègrent différents traits instrumentaux ou même interprétatifs. Ces derniers devront cependant faire l'objet d'une triangulation rigoureuse à l'échelle de groupes d'acteurs ou bien de l'organisation dans son ensemble.

${ }^{9}$ Moins fréquente que la première sous-trajectoire si l'on se base sur la littérature structurationniste.
} 
chir la porte G1. Les trois cas développés par Geoff Walsham (Walsham, 1993) notamment (l'« agence gouvernementale », «the processing company » et «the sky building society») illustrent que souvent les acteurs vont entrer dans des jeux subtils afin de maintenir le statu quo tout en utilisant, à leur façon, le nouveau système. Dans certains cas, on remarque que les usages ont des effets paradoxaux en contribuant à renforcer l'autonomie des acteurs alors que la technologie était destinée à développer le contrôle central. De la même manière, les études effectuées par Gudela Grote et Günter H. Baitsch (Grote et Baitsch, 1989), Frantz Rowe et Emmanuel Monod (Rowe et Monod, 2000) ou encore de F.-X. de Vaujany (Vaujany, 2001b) valorisent largement la dynamique équilibrée, avec d'éventuels passages de G1, mais de fréquent blocage en G2.

Dans le cas d'un nouveau système de messagerie mis en œuvre dans une entreprise du BTP, G. Grote et G.H. Baitsch constatèrent la présence de deux types de technologies-enpratique. La première était portée par les employés du service « construction ». Elle était plutôt centrée sur la tâche et un maintien du statu quo. La seconde était liée aux employés du service de « gestion immobilière » nouvellement créé dans le cadre d'une réorganisation. Elle était davantage centrée sur le contrôle et un changement du système de rôles. En effectuant une observation longitudinale, les chercheurs relevèrent tout d'abord que « le nouveau système était utilisé principalement pour des contacts orientés projets et beaucoup moins pour des contacts hiérarchiques, c'est-à-dire les contacts requis par l'organisation bureaucratique des compétences » (Grote et Baitsch, 1989, p. 135). Et en dépit de quelques patterns très locaux d'innovation sociale à l'usage (orientés tâche), d'une façon générale, le groupe construction avait reproduit dans ses usages le périmètre, les formes d'interaction et les identités réciproques à l'œuvre dans le système de rôles habituel. À l'inverse, pour le groupe de gestion immobilière, le nouveau système avait servi de support à une stratégie de contrôle des bureaux régionaux du groupe et de renfoncement du siège central. Malheureusement pour ce groupe, « [...] les bureaux régionaux ont résisté à l'intrusion par le biais du nouveau système en ne répondant pas » (p. 137) ou tout simplement en refusant d'être intégrés dans le périmètre technique du réseau. Finalement, pour les deux groupes (construction et immobilier), les trajectoires appropriatives sont restées bloquées en G1.

De la même manière, F. Rowe et E. Monod (Rowe et Monod, 2000) purent noter en France des dynamiques très neutres dans le milieu bancaire en matière de messagerie. Les auteurs remarquèrent qu'il y eut « adoption de la messagerie à la culture bancaire et non l'inverse » (p. 156). Globalement, la messagerie n'a pas modifié le périmètre et la structure du système de rôles, que ce soit au sein des agences ou dans les communications entre les agences et le siège.

Enfin plusieurs recherches (Vaujany, 2000a ; Vaujany, 2000b ; Vaujany, 2001b ; Newell et al., 2001) mirent également en lumière des dynamiques neutres voire perturbées en matière d'intranet. Contrairement à un discours managérial fort sur le pouvoir décloisonnant des intranets, ces études montrèrent que la technologie n'a finalement fait que reproduire et même renforcer ces mêmes aspects du système de rôles qu'elle était censée dépasser. Les sites développés comme ceux utilisés correspondaient aux frontières organisationnelles les plus fines.

Les différents exemples que nous venons de donner portent plutôt sur des systèmes de communication (type e-mail) ou des systèmes d'information que d'aucun pourront juger 
plutôt faiblement $«$ restrictifs ${ }^{10}$. Le lecteur peut donc légitimement se poser la question suivante : la dynamique du point d'équilibre, avec ses portes G1 et G2, ne s'applique-t-elle pas plutôt à des technologies présentant un faible niveau de restrictivité ? Après avoir précisé qu'une telle question n'est légitime que dans une perspective réaliste critique, il nous semble que la réponse à cette interrogation ne saurait être définitive. Le degré de restrictivité de la technologie a probablement une influence sur le rythme du déploiement de la «trajectoire appropriative », mais pas sur sa nature profonde. En d'autres termes, la technologie va probablement contraindre les processus, sans véritablement les déterminer. Le contre-exemple donné par certaines études françaises en matière de workflow ou de PGI va plutôt dans le sens de l'affirmation qui précède.

Étudiant des cas d'utilisation de PGI, Martine Carbonel (Carbonel, 2001) commence par noter le caractère plutôt restrictif de ce genre d' outils ${ }^{11}$. Mais si l'auteur insiste sur l'aspect « structurant» du système, plusieurs cas montrent comment des acteurs ont joué sur la technologie afin de l'insérer dans le système de rôles préexistant. À un niveau intermédiaire, les informaticiens locaux peuvent parfois « refuser le diktat du logiciel et développer des programmes spécifiques pour coller aux habitudes et respecter l'organisation habituelle » (p. 78). De la même manière, Gérard Valenduc (Valenduc, 2000) remarque la dimension très structurante des PGI, notamment au sein d'une université où cette technologie a impliqué un réaménagement des tâches dans les différents départements, mais il souligne aussi des jeux d'acteurs visant à faire tourner l'outil sans modifier le fonctionnement du système de rôles. Plusieurs monographies l'illustrent : en refusant d'alimenter le système du PGI ou bien en le renseignant de manière à maintenir l'ordre en place, les acteurs peuvent activer des inhibiteurs qui rendront problématique la porte $\mathrm{G} 1^{12}$.

De la même manière, David Muhlmann (Muhlmann, 2001) retrouve des dynamiques neutres par rapport à une technologie de workflow pensée pourtant de façon à renforcer le contrôle et à créer des dynamiques d'échanges inédites. Il constate ainsi que les commerciaux de la société étudiée se coordonnaient informellement avec les directeurs commerciaux (par téléphone) avant de saisir des informations sur le système. L'auteur arrive à la conclusion que «le processus d'informatisation s'enkyste toujours dans les construits sociaux préexistants et dans des rapports de pouvoir lui donnant sa forme, et les nouvelles

\footnotetext{
10 La restrictivité (cf. Desanctis et al., 1992 ; Desanctis et Poole, 1992) correspond à la capacité d'un nouveau système à contraindre les interactions avec l'utilisateur à un ensemble d'actions prédéfinies. Une technologie restrictive est illustrée par l'exemple du distributeur automatique de billets. Celui-ci présente une arborescence réduite, aucune possibilité de paramétrages directs et des modalités très simples d'interaction. En outre, de nombreux apprentissages sociaux (des films, des publicités, des retraits avec le papa...) rendent l'objet très univoque. À l'inverse, le cas des outils hautement équivoques peut être illustré par l'exemple des réseaux de neurones formels. Ces systèmes-experts offrent de nombreuses possibilités de paramétrages. Il peuvent être finalisés de multiples façons (prévision de chiffre d'affaires, défaillances d'entreprises, taux de croissance économique...). De plus, ils sont encore peu définis socialement, car plutôt neufs.

11 «Les capacités de configuration du progiciel déterminent le niveau de personnalisation de l'entreprise. Le plus souvent, le paramétrage est statique, peu polymorphe et évolue dans un espace fini » (Carbonel, 2001, p. 81).

12 Mais pour $\mathrm{G}$. Valenduc, la technologie semble finalement plus propice à des dynamiques régénérantes que neutres : « Le caractère structurant des ERP [PGI] reste cependant toujours présent. Il est sous-jacent à toutes les orientations et limite fortement les marges de manœuvre. La standardisation, la hiérarchisation, la centralisation, l'effet d'engrenage, prennent des formes assez semblables dans le cas de la firme chimique et de l'université » (Valenduc, 2000, p. 204). On peut objecter que les deux cas qu'il reprend correspondent à une assimilation

« réussie » de l'outil et que certains éléments de son récit sont typiques d'un processus de réappropriation.
} 
technologies sont formatées par les fonctionnements organisationnels dans lesquels elles sont encastrées » (p. 342). Les périmètres, formes d'interaction et identités du système de rôles restent inchangés par des usages qui consolident les positions de chacun.

Afin de répondre plus directement à la question soulevée par rapport au degré de restrictivité de la technologie, il semble cependant raisonnable de dire que les technologies les plus restrictives, dans la mesure où elles vont effectivement créer des dynamiques régénérées, vont le faire de façon plus rapide et cohérente sur le plan organisationnel que des technologies faiblement restrictives.

\subsubsection{La trajectoire improvisationnelle}

La seconde trajectoire appropriative correspond à des séquences archétypiques à la fois moins stabilisées et plus diffuses que pour une dynamique équilibrée. Dans le cas d'une dynamique improvisationnelle, la technologie est reconstruite socialement de façon récurrente. Les utilisateurs vont modifier les paramétrages, refinaliser l'outil et changer son insertion dans le système de rôles à des intervalles réguliers.

On pourrait définir la trajectoire improvisationnelle comparativement à la trajectoire équilibrée au moyen du tableau suivant :

Tableau 1

Comparaison dynamique équilibrée-dynamique improvisationnelle

\begin{tabular}{ccc}
\hline & Dynamique équilibrée & Dynamique improvisationnelle \\
\hline Séquence archétypique & N-P-R-N & R1-R2-R3 \\
Rythme & Faible & Rapide \\
Degré de cohérence & Élevé & Faible \\
\hline
\end{tabular}

La littérature structurationniste présente de nombreux cas qui exposent des situations d'évolutions sociotechniques des mois voire des années après l'implantation d'un système (cf. Orlikowski, 2000 ; Vaujany, 2001b). Une étude sur un grand groupe énergétique français notamment relève de véritables processus régénérants pour un outil de messagerie, pourtant parmi les technologies les plus anciennes de l'entreprise (Vaujany, 2001b ; Vaujany, 2001c).

\subsubsection{La trajectoire catalytique}

Cette dernière trajectoire est la plus intéressante, car largement problématique par rapport à la littérature structurationniste. Dans la perspective de cette dynamique, les usages jouent le rôle d'un catalyseur ou d'un inhibiteur par rapport à des processus qui se sont formés dans des champs extérieurs à la technologie. D'après Marie-Josée Avenier, la catalyse correspond à « l'accélération d'une transformation souhaitée par la présence, dans le contexte de cette transformation, d'un facteur, le catalyseur ${ }^{13}$ (Avenier, 1999, p. 13). Le catalyseur est donc un élément (objet matériel ou immatériel) qui va contribuer à l'accélération de certains processus sociaux. La logique de production-reproduction des structures sociales via les usages qui était centrale dans les deux trajectoires précédentes perd donc de

\footnotetext{
13 On remarque que cette vision du changement a des origines qui remontent probablement aux recherches de James B. Quinn (Quinn, 1977) sur les «precipitating events» pour les sciences de gestion. De nombreux autres travaux des sciences sociales ont également repris le principe de la catalyse, comme ceux de Sandra A. Waddock et James E. Post (Waddock et Post, 1991).
} 
sa pertinence. L'usage de la technologie ne correspond plus qu'à une catégorie d'actions parmi beaucoup d'autres. Les objets les plus structurants de l'organisation sont liés à des champs non technologiques.

Dans le cas de la mise en œuvre d'un intranet, il semble ainsi que l'outil n'a fait, pour certains individus, qu'accélérer des processus de décloisonnements initiés au moyen d'objets «classiques » de l'organisation : le téléphone, les rencontres directes, les e-mails (Vaujany, 2001b ; Vaujany, 2001c).

En conclusion de cette première partie, on peut légitimement se demander si ces différentes trajectoires constituent des horizons pratiquement et épistémologiquement irréconciliables. Il est vraisemblable que non. Tout va sans doute dépendre du terme de l'observation (court ou long), du niveau de l'étude (microsocial, organisationnel ou institutionnel) ainsi que du prisme théorique retenu (structurationnisme « pur » ou amendé). Il est d'ailleurs très possible, à des degrés divers, que les trois dynamiques puissent cohabiter au sein d'une même organisation.

\section{Logiques de contrôle et gestionnaires du changement sociotechnique}

Faut-il gérer et, si oui, comment gérer les dynamiques sociotechniques décrites précédemment ? C'est à cette double interrogation que nous allons nous efforcer de répondre maintenant en suggérant différentes « configurations » de gestion. S'inscrivant dans la mouvance du réalisme critique, ces configurations reprennent le modèle archétypique présenté dans la première partie.

\subsection{Facilitation, canalisation et sélection ou la difficile gestion du changement technique}

\subsubsection{Présentation des logiques de contrôle d'une trajectoire appropriative}

Le thème de la gestion des processus de structuration est rarement abordé dans le champ structurationniste, et lorsque cela est le cas, il l'est de façon non systématique. On peut cependant remarquer la présence de trois logiques de contrôle, basées sur les capacités plus ou moins forte d'auto-organisation des systèmes sociotechniques (Vaujany, 2001c).

La première logique de contrôle est axée sur la facilitation. Le concept apparaît dans de multiples travaux en sciences sociales. On pourrait citer notamment les recherches de Rosemary O'Leary et Susan Summers Raines (O'Leary et Summers Raines, 2001) en sciences politiques, Manoj Hastak et Anusree Mitra (Hastak et Mitra, 1996) en marketing, Phil Kirk et Mike Broussine (Kirk et Broussine, 2000) en gestion des ressources humaines, Terry L. Griffith (Griffith et al., 1998) en système d'information, Michael P. Hottenstein, Michael S. Casey et Steven C. Dunn (Hottenstein et al., 1997) ou John Farrell et Richard Weaver (Farrell et Weaver, 1998) en gestion de production, ou encore Terry A. Beehr et David C. Gilmore (Ferris et al., 1978) ou Wendy J. Gregory et Norma R. Romm (Gregory et Romm,

2001) en management général. Les définitions données par la suite seront concentrées sur deux champs disciplinaires : la gestion des ressources humaines et le système d'information.

En gestion des ressources humaines, le but de la facilitation est défini comme consistant à « établir ou maintenir un environnement au sein duquel l'apprentissage est créé » (Kirk et Broussine, 2000, p. 13). De façon proche, Terry Bentley explique aussi que le but de la facilitation va être de « fournir des opportunités pour l'apprentissage » (Bentley, 1994, p. 9). 
Dans le cas du système d'information, la facilitation est définie comme un « ensemble de facteurs ou d'actions accomplies avant, pendant ou après une réunion afin d'aider un groupe à atteindre ses objectifs » (Bostrom et al., 1993). Elle est aussi parfois définie comme « le fait de passer du connu à l'inconnu » (Killian et Simmons, 1992) et donc de mener des actions censées mettre en mouvement le système social ciblé sans pour autant avoir une idée précise de la «bonne » direction à atteindre. Sur ce dernier point, certains chercheurs adoptent une position de plus en plus critique. Stephen C. Hayne (Hayne, 1999) insiste ainsi sur le fait que si le rôle du facilitateur est souvent présenté comme s'inscrivant dans le cadre d'une « stricte impartialité », celui-ci va parfois « diriger et structurer les processus d'un groupe », consciemment ou inconsciemment. Ce constat est largement concordant avec celui effectué par T.L. Griffith, Mark A. Fuller et Gregory B. Northcraft (Griffith et al., 1998) en matière de systèmes d'aide à la décision de groupe. C'est un point qui ressort également dans les recherches d'autres champs disciplinaires, comme en gestion des ressources humaines avec P. Kirk et M. Broussine (Kirk et Broussine, 2000). Ces auteurs suggèrent d'ailleurs une typologie d'agents facilitateurs en fonction de leur degré de conscience politique et de la forme que celle-ci prend ${ }^{14}$. Dans le champ du système d'information, l'action de facilitation s'effectue plutôt à l'échelle de groupes et dans un contexte ponctuel (celui de réunion supportée par la technologie). Plusieurs études, comme celles de Fred Niederman (Niederman, 1997), Alan R. Dennis, Barbara H. Wixom et Robert J. Vandenberg (Dennis et al., 2001), ou encore Tan Bcy, Wei Kwok-Kee et John E. Lee-Partridge (Bcy et al., 1999) sont typiques de ce contexte facilitateur.

En résumé, on définira la facilitation comme correspondant à l'ensemble des actions qui visent à favoriser l'entrée d'un système sociotechnique donné dans l'archétype régénérant sans pour autant chercher à déterminer précisément la forme et le contenu que celui-ci revêtira. La facilitation a donc deux dimensions. Une première dimension, cognitive, qui permet d'insister sur les apprentissages que le facilitateur devra stimuler. Une seconde dimension porte davantage sur les aspects politiques. Il s'agira, conformément à l'idée de G. Walsham (Walsham, 1993), de créer un contexte favorable à un changement sociopolitique profond du système ciblé, de construire des « coalitions pour le changement ». On note que les dimensions cognitives et politiques sont largement interdépendantes, un changement struc- turel étant souvent indissociable de processus d'apprentissage de la part des acteurs.

La deuxième logique de contrôle sociotechnique, la canalisation, repose sur un principe plus coercitif que la facilitation. Elle regroupe l'ensemble des actions dont le but est d'orienter l'évolution d'un système sociotechnique vers une forme précise d'archétype régénéré. Ce second mode de gestion des processus de structuration apparaît également dans la littérature structurationniste. On le trouve le plus clairement dans la «théorie de la structuration adaptative » (Desanctis et Poole, 1990 ; Desanctis et Poole, 1992 ; Desanctis et Poole, 1994). Les auteurs suggèrent d'adopter, parfois, un niveau élevé de restrictivité et de présenter une vision claire et active de la finalité globale du système, son « esprit ». Les auteurs pensent ainsi qu' « un esprit cohérent canalise l'utilisation de la technologie dans des directions définies. On s'attendra à l'inverse à ce qu'un esprit incohérent exerce une influence plus faible sur le comportement des utilisateurs » (Desanctis et Poole, 1994,

\footnotetext{
14 « Conscience-fermée partielle», « conscience immobilisée», « conscience manipulatrice» et « conscience ouverte partielle» (Kirk et Broussine, 2000, pp. 17-22).
} 
p. 127). Certains auteurs non structurationnistes soulignent aussi la nécessité d'entreprendre, ponctuellement, des actions coercitives en matière de gestion des appropriations, notamment dans une situation de dérapage comme celle relevée par Nicolas Carr (Carr, 1999).

La dernière et troisième logique de contrôle repose sur un principe de sélection. Cette logique regroupe l'ensemble des actions correspondant à une gestion catalytique d'un système sociotechnique. Elle vise seulement à renforcer ou affaiblir certains processus de structuration. L'objectif de l'agent catalysant va ainsi être d' " identifier les inhibiteurs du développement des comportements souhaités et de repérer ses catalyseurs potentiels, afin de tenter d'inhiber les uns et de tirer parti des autres » (Avenier, 1999, p. 23). On note que plusieurs travaux ne font pas de distinction toujours très nette entre catalyse et facilitation. C'est le cas de la recherche menée par M. Hastak et A. Mitra (Hastak et Mitra, 1996) en marketing, où la facilitation est opposée à l'inhibition de processus. La facilitation correspond cependant à un type d'action différent du cadre de la définition donnée précédemment. En effet, un ensemble de formes pertinentes est envisagé par les gestionnaires qui facilitent l'action, ce qui est contraire à l'esprit de la facilitation ${ }^{15}$. En outre, le principe de gestion décrit (jeu opportuniste sur des inhibiteurs ou des catalyseurs) est davantage conforme à un mode de gestion catalytique que facilitateur.

\subsubsection{Les FCP (facteurs critiques perçus) ou l'évaluation des logiques de contrôle}

Comment contextualiser les logiques de contrôle ? Quels points de repère donner aux agents de changements qui s'efforcent de piloter le processus (qu'ils soient facilitateurs, canalisateurs ou encore catalyseurs) ? L'idée d'une qualité variable des usages est relativement fréquente dans le champ structurationniste. G. Walsham et Chun-Kwong Han (Walsham et Han, 1993) parlent d' «efficacité à l'usage », W.J. Orlikowski et al. (Orlikowski et al., 1995) d' « efficacité des usages », G. Desanctis et M.S. Poole (Desanctis et Poole, 1994) de « qualité » et d' " efficience » en matière de décisions supportées par un système d'aide à la décision de groupe. Afin d'évaluer globalement la valeur à l'usage d'un nouvel outil, les FCP peuvent être utiles.

La notion de FCS (facteurs-clés de succès) est d'un usage ancien en système d'information. Elle remonte aux premiers travaux de Terry Daniel (Daniel, 1961) et John F. Rockart (Rockart, 1979), même si les propositions de ce dernier portaient plus spécifiquement sur les éléments qui favorisent une implémentation réussie du système d'information et non ceux liés à la réussite de l'organisation en général. L'expression a connu depuis une diffusion importante au sein des différentes sous-disciplines de la gestion, allant du système d'information (Lincoln, 1991) à la stratégie (Leidecker et Bruno, 1984 ; Calori et Attamer, 1993). D'après Gérard Koenig, un FCS désigne un « élément considéré comme un gage de réussite à une période donnée de l'histoire du secteur ${ }^{16}$ (Koenig, 1996, p. 530). En

\footnotetext{
15 Même si la facilitation peut inclure des aspects politiques dont le facilitateur ne sera pas forcément conscient et qu'il gagnera à expliciter à lui-même afin de mieux les maîtriser (Kirk et Broussine, 2000).

16 On note qu'aujourd'hui le concept est devenu largement polysémique dans son appropriation par les managers (Ketelhohn, 1998). Certains auteurs appliquent ainsi le concept aux facteurs qui favorisent l'assimilation réussie d'une technologie, comme Henk Akkermans et Karl Van Helden (Akkermans et Van Helden, 2002) en matière de PGI ou à la définition de profils pertinents de cadres SI comme J.F. Rockart (Rockart, 1982).
} 
système d'information, le concept est utilisé aussi bien dans la démarche de planification du système d'information (Shank et al., 1985 ; Lincoln, 1991), pour la détermination des informations critiques que devra alimenter le système d'information (Crescenzi et Reck, 1985) ou encore l'évaluation postimplémentation d'un nouvel outil (Cerullo, 1982 ; Symons et Walsham, 1987). La notion de FCS est cependant trop globale et positive pour coller au positionnement épistémologique du cadre théorique exposé dans la première partie. C'est pourquoi la notion de FCP lui sera préférée dans la suite de l'article. Un FCP est « un élément (partie du système d'offre ou processus organisationnel) qui est rapporté comme fondamental par une catégorie spécifique de parties prenantes pour la survie de l'organisation et son succès dans le long terme » (Vaujany, 2001c, p. 121). Afin maintenant d'articuler logiques de contrôle, FCP et trajectoires appropriatives, on pourrait avancer les quatre configurations de gestion suivantes, basées sur un double principe de cohérence interne et externe :

Tableau 2

Trois configurations de gestion des trajectoires appropriatives

\begin{tabular}{|c|c|c|c|c|}
\hline & Caractéristiques & $\begin{array}{c}\text { Référence } \\
\text { à des FCP ? }\end{array}$ & $\begin{array}{c}\text { Contexte } \\
\text { appropriatif }\end{array}$ & Exemples d'actions \\
\hline Facilitation & $\begin{array}{l}\text { Gestion indirecte et ouverte } \\
\text { des trajectoires } \\
\text { appropriatives. Le système est } \\
\text { censé avoir un degré élevé } \\
\text { d'auto-organisation. } \\
\text { L'ensemble n'est pas } \\
\text { véritablement dominé par des } \\
\text { parties prenantes précises, ou } \\
\text { si c'est le cas, celles-ci } \\
\text { présentent une faible } \\
\text { légitimité générale. La valeur } \\
\text { de l'outil est à lier } \\
\text { directement au processus } \\
\text { d'appropriation et aux } \\
\text { « bricolages » des acteurs. }\end{array}$ & Non & $\begin{array}{l}\text { Dynamique } \\
\text { improvisationnelle. } \\
\text { La technologie joue } \\
\text { un rôle significatif } \\
\text { et continu dans } \\
\text { l'évolution du } \\
\text { système } \\
\text { sociotechnique. }\end{array}$ & $\begin{array}{l}\text { Formations en salle } \\
\text { ou itinérantes, } \\
\text { méthodes } \\
\text { d'autoformation, } \\
\text { ouverture du } \\
\text { paramétrage aux } \\
\text { utilisateurs, } \\
\text { accessibilité } \\
\text { importante, création } \\
\text { d'espaces } \\
\text { transversaux, } \\
\text { interventions } \\
\text { d'agents } \\
\text { facilitateurs. }\end{array}$ \\
\hline Sélection & $\begin{array}{l}\text { Gestion sélective, } \\
\text { ponctuelle et indirecte des } \\
\text { trajectoires appropriatives. } \\
\text { Le système sociotechnique, } \\
\text { autorégulé, est dominé par } \\
\text { des parties prenantes } \\
\text { précises qui restent } \\
\text { relativement en retrait. La } \\
\text { valeur des outils est à lier } \\
\text { directement aux usages des } \\
\text { acteurs, parfois stimulés par } \\
\text { les gestionnaires du système } \\
\text { d'information. }\end{array}$ & Oui & $\begin{array}{l}\text { Dynamique } \\
\text { catalytique. La } \\
\text { technologie joue un } \\
\text { rôle accessoire mais } \\
\text { réel dans l'évolution } \\
\text { du système } \\
\text { sociotechnique. }\end{array}$ & $\begin{array}{l}\text { Gestion par émission } \\
\text { de signes forts, } \\
\text { communication } \\
\text { renforcée sur les } \\
\text { usages pertinents, } \\
\text { sites ou forums } \\
\text { « best practices », } \\
\text { recours à des } \\
\text { facilitateurs politisés. }\end{array}$ \\
\hline
\end{tabular}




\begin{tabular}{|c|c|c|c|c|}
\hline & Caractéristiques & $\begin{array}{l}\text { Référence } \\
\text { à des FCP? }\end{array}$ & $\begin{array}{c}\text { Contexte } \\
\text { appropriatif }\end{array}$ & Exemples d'actions \\
\hline Canalisation & $\begin{array}{l}\text { Gestion directe, incitative et } \\
\text { continue des trajectoires } \\
\text { appropriatives. Le système } \\
\text { est majoritairement } \\
\text { hétéro-régulé. Sa cohésion } \\
\text { repose sur l'action d'une } \\
\text { régulation de contrôle } \\
\text { légitime. La valeur des } \\
\text { outils est ici davantage liée } \\
\text { à leur architecture et leur } \\
\text { utilité informationnelle } \\
\text { qu'aux usages proprement } \\
\text { dits. Les gestionnaires vont } \\
\text { s'efforcer de rapprocher le } \\
\text { système de rôles de } \\
\text { l'organisation de celui pensé } \\
\text { pour le nouveau système. }\end{array}$ & Oui & $\begin{array}{l}\text { Dynamique du } \\
\text { point d'équilibre. } \\
\text { La technologie joue } \\
\text { un rôle significatif } \\
\text { et alternatif dans } \\
\text { l'évolution du } \\
\text { système } \\
\text { sociotechnique. }\end{array}$ & $\begin{array}{l}\text { Développement de } \\
\text { systèmes } \\
\text { d'incitation, } \\
\text { interventions } \\
\text { directes de leaders } \\
\text { d'appropriation, } \\
\text { définition de niveau } \\
\text { d'accessibilité } \\
\text { rigoureux, } \\
\text { possibilités de } \\
\text { paramétrage étroites, } \\
\text { communication } \\
\text { intensive et ciblée } \\
\text { sur les objectifs du } \\
\text { système. }\end{array}$ \\
\hline
\end{tabular}

Les différents cas élaborés par les structurationnistes montrent qu'afin d'éviter des tensions au sein du système sociotechnique et de développer des blocages du type G2, une mise en cohérence des logiques de contrôle avec les trajectoires appropriatives, le contexte organisationnel et les éventuels FCP, est nécessaire. À un système sociotechnique instable, fortement différencié et plutôt auto-organisé devrait être associée une logique de contrôle basée essentiellement sur la facilitation, c'est-à-dire sur des actions souples et ouvertes. Inversement, pour un système sociotechnique stable, inerte, plutôt homogène et hétéroorganisé, des logiques de contrôle plus directives devraient être déployées. L'hypothèse sous-jacente est que le changement est un des buts principaux de l'implémentation technologique, et que les FCP, s'ils peuvent être déterminés ${ }^{17}$, serviront alors de ligne de mire à l'évaluation.

Interviennent à ce stade trois figures concrètes de la gestion du changement sociotechnique liées aux trois logiques de contrôle : les agents facilitateurs ${ }^{18}$, les agents canalisateurs et catalyseurs. On pourrait les présenter au moyen du tableau suivant :

\footnotetext{
17 Dans le cas d'une entité auto-organisée où sont présents surtout des agents primaires (c'est-à-dire une agrégation d'individus sans projets collectifs et avec des ressources faibles) et quelques agents institués (un regroupement d'agents ayant un objectif commun et des ressources importantes) pour reprendre la distinction proposée par M.S. Archer (Archer, 1995). La détermination des FCP sera alors problématique. En effet, les FCP supposant l'existence d'un point de vue focal réel et homogène, leur élaboration ne sera pas possible dans ce contexte social « dilué ».

18 Pour le cas du facilitateur, divers travaux, comme ceux de F. Niederman (Niederman, 1997) traitent de l'influence des variables nationales sur leur action. Peu de recherches ont abordé les autres figures sous l'angle multiculturel.
} 
Tableau 3

Les figures de la gestion du changement sociotechnique

\begin{tabular}{lll}
\hline & \multicolumn{1}{c}{ Caractéristiques } & \multicolumn{1}{c}{ Qualités requises } \\
\hline Facilitateur & - Animateur dégagé & - Empathique \\
& - Formateur & - Consensuel \\
& - Agent apolitique & - Réflexif \\
\hline Canalisateur & - Guide engagé & - Bonne culture technique \\
& - S'efforce d'orienter l'action vers des & - Charismatique \\
& buts prédéfinis & - Vision globale des enjeux et \\
& & articulations des processus sociaux avec les \\
\hline \multirow{4}{*}{ Catalyseur } & - Agent politique & - Bonne culture technique \\
& - Accompagnateur & - Bonne connaissance du système de valeur \\
& - Joue sur les inhibiteurs ou & de l'organisation cible \\
& catalyseurs d'actions qui sont jugées & - Bon communicant et gestionnaire de signe \\
& pertinentes & - Sens de l'opportunisme \\
& - Suit les dynamiques & - Bonne culture technique \\
\hline & Sociotechniques davantage qu'il ne les génère &
\end{tabular}

Ces différentes figures déclinent en fait des rôles qui peuvent être endossés par les mêmes acteurs à un même moment. De plus, ces personnes impliquées en continu ou à temps partiel dans l'accompagnement d'un système sociotechnique pourront aussi s'appuyer dans leur travail sur deux classes d'acteurs informels : les agents «métastructurants » et les agents imitateurs.

Les processus de métastructuration ont été relevés par W.J. Orlikowski et al. (Orlikowski et al., 1995). Ils regroupent toutes les actions de médiation des usages qui correspondent à des dynamiques de structuration de second ordre, c'est-à-dire des processus de structuration contraignant ou habilitant d'autres dynamiques de structuration. Les agents métastructurants sont ainsi ces agents qui « interviennent régulièrement et délibérément dans la structuration des activités des utilisateurs en influençant leur compréhension, en modifiant les caractéristiques structurelles du système afin de le rendre plus facile à utiliser, en modifiant les pratiques institutionnelles, et en facilitant l'accès et l'usage des technologies de communication » (p. 437). Pour W.J. Orlikowski, les agents métastructurants peuvent avoir un statut formel (rejoignant alors une des trois figures introduites précédemment) ou informel. Il s'agit alors de sortes de « leaders d'appropriation ». Concrètement, ces acteurs peuvent aller du jeune stagiaire issu d'une grande école qui fait son entrée au moment du lancement d'un nouveau système, en passant par le contrôleur de gestion passionné d'informatique, jusqu' au responsable de projet sensible aux nouvelles technologies.

La deuxième classe d'agents, les imitateurs, apparaissent aussi bien dans la littérature structurationniste que dans le courant institutionnaliste. Ces acteurs vont repérer des technologies-en-pratique qui leur paraîtront pertinentes pour leur travail avant de les reproduire. Ils jouent ainsi un rôle central dans la diffusion d'innovations sociales à l'usage au sein du système de rôles ou dans la consolidation des structures sociales en place.

La bonne connaissance des deux figures informelles est fondamentale pour les agents formels, ceux qui sont investis plus ou moins de façon continue par la hiérarchie dans la gestion du changement sociotechnique. Le gestionnaire d'un nouveau système pourra s'appuyer sur des leaders d'appropriation ou activer les processus d'imitation en fonction des dynamiques sociales qu'il constatera. 


\subsection{Conclusion : limites et perspectives d'une gestion centrée sur les logiques de contrôle appropriatif}

Quelles sont les limites des configurations de gestion développées précédemment ainsi que des logiques de contrôle ou figures qu'elles intègrent? Nous pensons qu'elles sont de deux ordres : pratique, d'une part, et méthodologique, d'autre part.

La limite pratique est finalement dans le message même dont est porteuse la grille d'analyse. Conformément au positionnement non déterministe du modèle archétypique, les configurations de gestion invitent surtout à une gestion ex post des dynamiques sociales. C'est au fil des usages que va s'effectuer le pilotage. En phase de conception, la grille d'analyse et sa philosophie sous-jacente suggèrent à la rigueur d'implanter l'outil le plus modulaire possible. La phase de lancement sera plutôt accompagnée par des actions de facilitation. Ensuite, en fonction des appropriations et de leur degré de cohérence avec d'éventuels FCP, différentes logiques de contrôle pourront être déployées. Globalement, la phase ex ante, celle de l'élaboration du système, n'est que rarement traitée par le courant structurationniste $^{19}$. Croire cependant que la valeur d'un outil se construit uniquement au fil des usages est aussi illusoire que d'affirmer que toute sa valeur est déterminée par la seule phase de conception. Pour les structurationnistes ${ }^{20}$, le travail d'élaboration d'un nouveau système fixe une part importante du champ des possibles de l'utilisation. Mais comme nous avons eu l'occasion de le souligner dans la première partie, même dans le cas des technologies les plus restrictives, les acteurs pourront donner une flexibilité sociale au système allant au-delà du « potentiel » technique stricto sensu. Pour finir cette première discussion sur les aspects pratiques d'une gestion des appropriations et son articulation avec les méthodes de conception ou de planification du système d'information ${ }^{21}$, on peut dire que le champ d'action des configurations concerne par définition la « technologie-enpratique ». Elle peut donc être éloignée de l'intention et des projets du concepteur, mettant cependant en cohérence du point de vue des utilisateurs l'enchevêtrement technologique que deviennent de nombreuses firmes. Planification, conception et accompagnement de la vie d'un nouvel outil sont des activités interdépendantes mais largement indéterminées dans une perspective structurationniste.

Que dire maintenant des limites méthodologiques à l'application des configurations ? Cet aspect constitue sûrement un agenda de recherche crucial. Deux difficultés doivent être surmontées. La première réside dans l'évaluation du degré de proximité entre une trajectoire concrète et une trajectoire théorique. La seconde porte sur l'appréciation de la cohérence entre FCP et trajectoires ou logiques de contrôle et trajectoires. Sur ces deux points, un travail quantitatif longitudinal est en cours. Il s'appuie sur l'utilisation d'échelles permettant d'évaluer le degré de perturbation, régénération ou neutralité d'un système sociotechnique ainsi que sur différents calculs de distances euclidiennes entre des processus théoriques (les trajectoires) et des processus réels.

Le thème du changement sociotechnique, passionnant, est loin d'être épuisé. Alimenté par les évolutions conjointes des organisations et des systèmes informatiques, le sujet

\footnotetext{
19 D'un point de vue managérial.

20 Surtout pour les « réalistes critiques ».

21 Cf. (Lincoln, 1991).
} 
connaît même un fort regain d'intérêt. C'est ainsi que la recherche en sciences sociales développe des approches de plus en plus processuelles afin de mieux appréhender la vie sociale liée à des technologies de réseau aujourd'hui omniprésentes dans les organisations. Parce qu'elle amène à se poser des questions fondamentales ${ }^{22}$, parce qu'elle suggère une modélisation des organisations sous forme de systèmes de rôles évolutifs, ou encore, parce qu'elle permet de rendre intelligibles des systèmes de plus en plus « distribués », la perspective structurationniste peut apporter un éclairage précieux aux chercheurs et aux praticiens qu'intéressent les problématiques sociotechniques.

\section{Glossaire}

Archétype sociotechnique : image stylisée d'une situation de changement sociotechnique. Plus précisément, l'archétype désigne un état structurationnel, une configuration sociotechnique durable décrite au travers de dimensions structurantes. Trois archétypes sont envisagés dans le modèle archétypique : neutre $(\mathrm{N})$, régénéré $(\mathrm{R})$ ou perturbé $(\mathrm{P})$.

Cohérence archétypique : degré d'homogénéité, pour un système sociotechnique donné (une administration, entreprise, un service, un groupe de projet...), des dynamiques d'usage. Le problème est le suivant : le système contient-il de multiples trajectoires appropriatives ou est-il suffisamment homogène pour être considéré comme un tout ?

Facteurs critiques perçus : un élément (partie du système d'offre ou processus organisationnel) qui est rapporté comme fondamental par une catégorie spécifique de parties prenantes pour la survie de l'organisation et son succès dans le long terme (exemple : les délais de livraison).

Modèle archétypique : modèle d'inspiration structurationniste qui formalise les changements sociotechniques liés à la mise en œuvre d'une innovation informatique et qui donne des points de repères aux gestionnaires pour accompagner ces changements. Il est constitué des différentes situations basiques auxquelles peut être confrontée une organisation et des enchaînements possibles de ces cas de figure (les trajectoires).

Porte symbolique : point de passage entre deux archétypes sociotechniques où vont entrer en jeu des aspects cognitifs, psychologiques ou politiques qui vont souvent freiner le déploiement d'une trajectoire. Il s'agit d'une sorte de « zone de transit » entre la routine et le changement, la routine et la perturbation ou encore la perturbation et une situation de changement sereine.

Restrictivité : degré d'ouverture d'un outil technique aux interventions de l'utilisateur. Gerardine Desanctis et Marshall S. Poole (Desanctis et Poole, 1994) envisagent deux dimensions à la restrictivité : technique (c'est alors les paramétrages et l'arborescence plus ou moins large du logiciel qui déterminent sa restrictivité), ou procédurale (c'est l'environnement social qui est alors déterminant). Dans le cas d'une interface de distributeur automatique de billets, l'outil est hautement restrictif. D'un point de vue technique, les paramétrages ouverts à l'utilisateur sont peu nombreux et l'arborescence est limitée. D'un

\footnotetext{
22 Qu'est-ce qu'un véritable changement sociotechnique ? Comment passe-t-on d'une situation de routine à une situation d'innovation sociale à l'usage ? Quels critères d'évaluation et quel point de vue adopter ? (Cf. 1.1. et la comparaison Giddens-Archer.)
} 
point de vue procédural, l'outil est faiblement équivoque. Un long apprentissage social (l'enfant qui suit son papa au distributeur, des films, des publicités...) donne clairement un but au support. À l'inverse, dans le cas d'un logiciel de réseau de neurones formels la restrictivité est plutôt faible. Les possibilités de paramétrages sont multiples, les applications nombreuses, le sens social encore instable en raison de la nouveauté de l'outil... les restrictivités techniques et procédurales sont donc faibles.

Rythme du changement archétypique : vitesse du passage d'un archétype à un autre dans le cadre de l'étude d'une trajectoire. Le rythme du changement archétypique est relatif aux enchaînements précédents (sur la même organisation) ou à d'autres contextes sociotechniques proches.

Séquence archétypique : enchaînement précis d'archétypes permettant de reconstituer une trajectoire technologique (exemple : N-P-R-N).

Structurationnisme : courant de recherche en système d'information né dans les années 1980, notamment avec les travaux de Stephen R. Barley (Barley, 1986). Il propose une vision des rapports entre technologie et société et s'inspire des travaux de sociologues comme Anthony Giddens, Margaret S. Archer, Peter Berger et Thomas Luckman ou encore Roy Bhaskar.

Trajectoire appropriative : enchaînement régulier d'archétypes technologiques. La notion de trajectoire permet d'introduire la dynamique dans le modèle archétypique et de saisir dans le temps les étapes que peut connaître un système sociotechnique avant de connaître un changement profond de ses structures. Trois trajectoires sont intégrées dans la version finale du modèle archétypique : la trajectoire équilibrée (où l'innovation technique finit souvent par se dissoudre dans le système de rôles traditionnel de l'organisation), la trajectoire improvisationnelle (où au contraire l'innovation technique sert de support continu à des évolutions plus ou moins majeures du système) et la trajectoire catalytique (où l'innovation ne fait qu'inhiber ou accélérer des changements initiés hors champ technologique).

\section{Références}

Akkermans, H., Van Helden, K., 2002. Vicious and Virtuous Cycles in ERP Implementation: A Case Study of Interrelations Between Critical Success Factors. European Journal of Information Systems 11 (1), 35-46. Archer, M.S., 1982. Morphogenesis Versus Structuration: On Combining Structure and Action. The British

Journal of Sociology 33 (4), 455-483.

Archer, M.S., 1995. Realistic Social Theory: The Morphogenetic Approach. Cambridge University Press, Cambridge.

Avenier, M.J., 1999. Intermédiation et catalyse : susciter des comportements inhabituels au sein d'une organisation. Papier de recherche 17, Euristik, UPRES-A CNRS 5055.

Barley, S.R., 1986. Technology as an Occasion for Structuring: Evidence from Observations of CT Scanners and The Social Order of Radiology Departments. Administrative Science Quarterly 31, 78-108.

Barley, S.R., 1990. The Alignment of Technology and Structure Through Roles and Networks. Administrative Science Quarterly 35, 61-103.

Bcy, T., Kwok-Kee, W., Lee-Partridge, J.E., 1999. Effects of Facilitation and Leadership on Meeting Outcomes in a Group Support System Environment. European Journal of Information System 8 (4), 233-246.

Bentley, T., 1994. Facilitation: Providing Opportunities for Learning. Journal of European Indistrial Training 18 (5), 8-22.

Bhaskar, R., 1989. The Possibility of Naturalism. Harvester, London $\left(1^{\text {st }} \quad\right.$ ed.,

1979). 
Bostrom, R., Anson, R., Clawson, V., 1993. Group Facilitation and Group Support Systems. In: Jessup, L., Valacich, J. (Eds.), Group Support Systems: A New Frontier. MacMillian, New York, pp. 146-168.

Bouchikhi, H., 1990. Structuration des organisations. Economica, Paris.

Calori, R., Atamer, T., 1993. Diagnostic et décision stratégiques. Dunod, Paris.

Carbonel, M., 2001. Dérives organisationnelles dans les projets ERP : les cas de Guerbet et de Gaumont. Revue système d'information et management 6 (1), 71-85.

Carr, N., 1999. The Politics of E-Mail. Harvard Business Review, March-April 1998, 12-13.

Cerullo, M.J., 1982. Post-Implementation Evaluation of Computer Systems. CPA 52 (5), 45-51.

Cézard, M., Gollac, M., Rougerie, C., 2000. L'ordinateur outil de travail et bien culturel. Actes de la recherche en sciences sociales $134,131-152$.

Ciborra, C.U., 1997. De Profundis? Deconstructing the Concept of Strategic Alignement. IRIS 20.

Ciborra, C.U., 1999. A Theory of Information Systems Based on Improvisation. In: Currie, W.L., Galliers, B. (Eds.), Rethinking Management Information Systems. Oxford University Press, Oxford, pp. 136-155.

Ciborra, C.U., 2001. Moods, Situated Action and Time: A New Study of Improvisation. IRIS 24.

Crescenzi, A.D., Reck, R.H., 1985. Critical Success Factors: Helping IS Managers Pinpoint Information Needs. Infosystems 32 (7), 52-54.

Daniel, R.D., 1961. Management Information Crisis. Harvard Buisness Review 39, September-October, 111-121.

Dennis, A.R., Wixom, B.H., Vandenberg, R.J., 2001. Understanding Fit and Appropriation Effects in

Group

Support Systems Via Meta-Analysis. MIS Quarterly 25 (2), 167-193.

Desanctis, G., Poole, M.S., 1990. Understanding the Use of Group Decision Support Systems: The Theory of Adaptative Structuration. In: Steinfield, C., Fulk, J. (Eds.), Theoretical Perspectives on Organization and New Information Technologies. Sage Publications, Inc.

Desanctis, G., Poole, M.S., 1992. Microlevel Structuration in Computer-Supported Group Decision Making. Human Communication Research 19 (1), September, 5-49.

Desanctis, G., Poole, M.S., 1994. Capturing the Complexity in Advanced Technology Use: Adaptative Structuration Theory. Organization Science 5 (2), May, 121-146.

Desanctis, G., Dickson, G.W., Jackson, B.M., Poole, M.S., 1992. Interpretative Analysis of Team Use of Group Technologies. Journal of Organizational Computing 3, 1-29.

Dobson, P.J., 1999. Approaches to Theory Use in Interpretive Case Studies. $10^{\text {th }}$ Australian Conference on Information Systems, Wellington, New Zealand, 1-3 December.

Dobson, P.J., 2002. Critical Realism and Information Systems Research: Why Bother With Philosophy? http: //informationR.net/ir/7-2/paper124.html.

El Haddad, N., 1987. Système d'information dans l'organisation. Approche interprétative. Étude de cas dans les cliniques. Thèse de doctorat en sciences de gestion, université des sciences sociales de Grenoble.

Emery, E., Trist, E., 1969. Socio-Technical Systems, in Emery Systems Thinking. Penguin Books, London.

Emery, E., Trist, E., 1973. Towards a Social Ecology. Plenum, New York.

Farrell, J., Weaver, R., 1998. Facilitation: A Formula For Success. The Journal for Quality and Participation 21 (5), 44-48.

Ferris, G.R., Beehr, T.A., Gilmore, D.C., 1978. Social Facilitation: a Review and Alternative Conceptual Model. The Academy of Management Review 3 (2), 338-348.

Fung, P., Lea, M., O'Shea, T., 1995. Constructing the Network Organization: Content and Context in the Developpement of Electronic Commerce. Organization Science 6 (4), 462-478.

Giddens, A., 1979. Central Problems in Social Theory. Macmillian, New York.

Giddens, A., 1984. The Constitution of Society: Outline of a Theory of Structuration. California University Press.

Goffman, E., 1967. Interaction Ritual, Essays on Face-to-Face Behaviors. Pantheon Books, New York.

Gregory, W.J., Romm, N.R., 2001. Critical Facilitation: Learning Through Intervention in Group Processes. Management Learning 32 (4), 453-467.

Griffith, T.L., Fuller, M.A., Northcraft, G.B., 1998. Facilitator Influence in Group Support Systems: Intended and Unintended Effects. Information System Research 9 (1), 20-36.

Grote, G., Baitsch, G.H., 1989. A Study of the Reciprocal Effects between Organizational Culture and the Introduction and Use of an Office Communication System. Actes du colloque Information culture et société : la montée des réseaux, mai. Éditeur Réseau TNS, Grenoble, pp. 127-138.

Hastak, M., Mitra, A., 1996. Facilitating and Inhibiting Effects of Brand Cues on Recall, Consideration Set, and Choice. Journal of Business Research 37 (2), 121-127. 
Hayne, S.C., 1999. The Facilitators Perspective on Meetings and Implications for Group Support Systems Design. Database for Advances in Information Systems 30, 72-91.

Hodgson, G., 1999. Structures and Institutions: Reflections on Institutionalism, Structuration Theory and Critical Realism. Workshop on Realism and Economics, 19, January.

Hottenstein, M.P., Casey, M.S., Dunn, S.C., 1997. Facilitation of Advanced Manufacturing Technology: Implementation and Transfer. Industrial Management, September-October, 8-12.

Jacques, E., 1957. The Changing Culture of e Factory. Routledge and Kegan Paul, London.

Jones, M., 1999. Structuration Theory. In: Currie, W.L., Galliers, B. (Eds.), Rethinking Management Information Systems. Oxford University Press, New York, pp. 103-134.

Jong-Young, K., 2000. Social Interaction in Computer-Mediated Communication. American Society for Information Science. Bulletin of the American Society for Information Science 26 (3), 15-17.

Ketelhohn, W., 1998. What is a Key Success Factor? European Management Journal 16 (3), 335-340.

Killian, J., Simmons, L., 1992. The Zen of Facilitation. Journal of Staff Developpement 13 (3), 2-5.

Kirk, P., Broussine, M., 2000. The Politics of Facilitation. Journal of Workplace Learning 12 (1), 13-22.

Koenig, G., 1996. Management stratégique. Dunod, Paris.

Legrenzi, C., 1997. Une étude empirique des travailleurs de l'information. L'informatique professionnelle 159, 3-16.

Leidecker, J.K., Bruno, A.V., 1984. Identifying and Using Critical Success Factors. Long Range Planning 17 (1), 23-33.

Lincoln, T., 1991. Managing Information Systems for Profit. John Wiley, Chichester.

Mingers, J., 2000. The Contribution of Critical Realism as an Underpinning Philosophy for OR/MS and Systems. Journal of Operational Research and Society 51 (11), 1256-1270.

Muhlmann, D., 2001. Des nouvelles technologies à l'image des vieilles organisations. Sociologie du travail 43 (3), $327-347$.

Mumford, E., 1999. Routinization, Re-engineering and Socio-Technical Design, Changing Ideas of the Organization of Work. In: Currie, W.L., Galliers, B. (Eds.), Rethinking management information systems. Oxford University Press, New York, pp. 28-44.

Newell, S., Scarbrough, H., Swan, J., 2001. From Global Knowledge Management to Internal Electronic Fences: Contradictory Outcomes of Intranet Developpement. British Journal of Management 12 (2), 97-111.

Niederman, F., 1997. Facilitating Computer-Supported Meetings: an Exploratory Comparison of US and Mexican Facilitators. Journal of Global Information Management 5 (1), 17-26.

O'Leary, R., Summers Raines, S., 2001. Lessons Learned From two Decades of Alternative Disputes Resolution Programs and Processes at the US Environmental Protection Agency. Public Administration Review 61 (6), 682-692.

Orlikowski, W.J., 1992. The Duality of Technology: Rethinking The Concept of Technology in Organizations. Organization Science 3 (3), 398-427.

Orlikowski, W.J., 2000. Using Technology and Constituting Structures: a Practice Lens for Studying Technology in Organizations. Organization Science 11 (4), 404-428.

Orlikowski, W.J., Robey, D., 1991. Information Technology and the Structuring of Organizations. Information Systems Research 12 (2), 143-169.

Orlikowski, W.J., Yates, J., Okamura, K., Fujimoto, M., 1995. Shaping Electronic Communication: The Metastructuring of Technology in The Context of Use. Organization Science 6 (4), 423-444.

Pasmore, W., Francis, C., Haldeman, J., Shani, A., 1982. Sociotechnical Systems: A North American Reflection on Empirical Studies of the Seventies. Human Relations 35 (12), 1179-1204.

Piaget, J., 1947. La psychologie de l'intelligence. Armand Colin, Paris.

Piaget, J., 1977. La naissance de l'intelligence chez l'enfant. Delachaux et Niestlé, Lausanne ( $9^{\mathrm{e}}$ édition).

Quinn, J.B., 1977. Strategic Goals: Process and Politics. Sloan Management Review 19 (1), 21-37.

Rockart, J.F., 1979. Chief Executives Define Their Own Data Needs. Harvard Buisness Review 57 (2), 81-93.

Rockart, J.F., 1982. The Changing Role of the Information Systems Executive. Sloan Management Review 24 (1), 3-14.

Rowe, F., Monod, E., 2000. Limites structurelles et culturelles à l'usage de la messagerie dans les banques à réseau. Réseaux 104, 140-158.

Schanck, R.C., Abelson, R.P., 1977. Scripts, Plans, Goals and Understanding. NJ Erlbaum, Hillsdale. 
Shani, A.B., Grant, R.M., Krishnan, R., Thompson, E., 1992. Advanced Manufacturing Systems and Organizational Choice: Sociotechnical Approach. California Management Review, Summer, 91-111.

Shank, M.E., Boynton, A.C., Zmud, R.W., 1985. Critical Success Factor Analysis as a Methodology for MIS Planning. MIS Quarterly 9 (2), 121-130.

Symons, V.J., Walsham, G., 1987. Evaluation of Information Systems: A social Perspective. Management Studies Research Paper 1, Cambridge.

Trist, E., Bamforth, K., 1951. Some Social and Psychological Consequences of Longwall Method of Coalgetting. Human Relations 4, 3-38.

Valenduc, G., 2000. Les PGI. Une technologie structurante ? Réseaux 104, 186-205.

Vaujany (de), F.X., 1999. Stylisation de l'appropriation individuelle des technologies Internet à partir de la TSA. Revue systèmes d'information et management 4 (1), 57-75.

Vaujany (de), F.X., 2000a. Usages d'un intranet et processus de structuration de l'organisation. Revue systèmes d'information et management 5 (2), 79-104.

Vaujany (de), F.X., 2000b. Technologie perturbatrice, technologie neutre et technologie régénérante : construction et approfondissement de trois archétypes technologiques. Actes du colloque de l'AIM, Montpellier. Actes sur CD-ROM.

Vaujany (de), F.X., 2001a. Grasping IT Use Social Dynamic: Illustration of a Structurational Approach. In: Remenyi, D., Brown, A. (Eds.), The Eight European Conference on Information Technology Evaluation. Edited by Proceedings of ECITE 2001, Oriel College, Oxford, pp. 325-334.

Vaujany (de), F.X., 2001b. American and European Social Embeddedness in IS Research: The Case of Structurational Approaches. Founding Conference of the European Academy of Management (EURAM). European Management Research, Trends and Challenges, Barcelona, 19-21 avril. Actes sur CD-ROM.

Vaujany (de), F.X., 2001c. Gérer l'innovation sociale à l'usage des technologies de l'information : une contribution structurationni ste. Thèse de doctorat en sciences de gestion, université Jean Moulin, Lyon 3.

Waddock, S.A., Post, J.E., 1991. Social Entrepreneurs and Catalytic Change. Public Administration Review 51 (5), 393-401.

Walsham, G., 1993. Interpreting Information Systems in Organisations. John Wiley, Chichester.

Walsham, G., Han, C.K., 1993. Information Systems Strategy Formation and Implementation: The Case of a Central Government Agency. Accounting Management and Information Technology 3 (3), 191-209. 